\title{
Millimeter Wave Power Transmission for Compact and Large-Area Wearable IoT Devices based on a Higher-Order Mode Wearable Antenna
}

\author{
Mahmoud Wagih, Member, IEEE, Geoffrey S. Hilton, \\ Alex S. Weddell, Member, IEEE, and Steve Beeby, Senior Member, IEEE
}

\begin{abstract}
Owing to the shorter wavelength in the millimeterwave (mmWave) spectrum, miniaturized antennas can receive power with a higher efficiency than UHF bands, promising sustainable mmWave-powered Internet of Things (IoT) devices. Nevertheless, the performance of a mmWave power receiver has not been compared, numerically or experimentally, to its sub$6 \mathrm{GHz}$ counterpart. In this paper, the performance of mmWavepowered receivers is evaluated based on a novel wearable textile-based higher-order mode microstrip antenna, showing the benefits of wireless power transmission (WPT). Firstly, a broadband antenna is proposed maintaining a stable wearable measured bandwidth from 24.9 to $31.1 \mathrm{GHz}$, over three-fold improvement compared to a conventional patch. The proposed antenna has a measured $8.2 \mathrm{dBi}$ co-polarized gain with the highest thickness-normalized efficiency of a wearable antenna. When evaluated for compact power receivers, the measured path gain shows that WPT at $26 \mathrm{GHz}$ outperforms $2.4 \mathrm{GHz}$ by $11 \mathrm{~dB}$. A rectenna array based on the proposed antenna is then evaluated analytically showing the potential for up to $6.3 \times$ higher power reception compared to a UHF patch, based on the proposed antenna's gain and an empirical path-loss model. Both use cases demonstrate that $\mathbf{m m W a v e - p o w e r e d ~ r e c t e n n a s ~ a r e ~ s u i t a b l e ~ f o r ~}$ area-constrained and large-area wearable IoT applications.
\end{abstract}

Index Terms-Antennas, Microstrip Antennas, MillimeterWave Antenna, Permittivity measurements, RF Energy Harvesting, Rectenna, Wireless Power Transfer

\section{INTRODUCTION}

$\mathbf{M}$ ILLIMETER WAVE bands have attracted significant interest for Internet of Things (IoT), Wireless Body Area Networks (WBAN), and sensing applications [1]-[7]. Textile-based millimeter-wave (mmWave) antennas have been demonstrated for various applications such as high data-rate communications [5], as well as detecting moving objects based on beam-switching arrays [3]. D. Pozar states that "At higher frequencies, more antenna gain can be obtained

This paragraph of the first footnote will contain the date on which you submitted your paper for review. This work was supported by the European Commission through the EnABLES Project, funded under H2020-EU.1.4.1.2 grant number: 730957, and the UK Engineering and Physical Sciences Research Council (EPSRC) under Grant EP/P010164/1, S. Beeby was supported by the UK Royal Academy of Engineering under the Chairs in Emerging Technologies scheme. (Corresponding author: M. Wagih)

M. Wagih, A. S. Weddell, and S. Beeby are with the School of Electronics and Computer Science, University of Southampton, Southampton, SO17 1BJ, U.K. (email: mahm1g15@ecs.soton.ac.uk)

G. S. Hilton is with the Communication Systems \& Networks Group, University of Bristol, Bristol BS8 1UB, U.K.

Copyright (c) 2021 IEEE. Personal use of this material is permitted However, permission to use this material for any other purposes must be obtained from the IEEE by sending a request to pubs-permissions@ieee.org for a given physical antenna size" [8]. This has motivated theoretical [9], [10] and practical [11], [12] investigations, in mmWave Wireless Power Transmission (WPT) for future IoT applications.

IoT WBANs have various applications in healthcare monitoring, fitness tracking, defense and wearable sensing [13], [14]. Powering such systems using microwave and mmWave rectennas has attracted significant research interest, from UltraHigh Frequency (UHF) to mmWave 5G bands [15]-[17], where wireless power transmission is increasingly seen as a reliable and scalable method for powering the IoT [18]. At mmWave bands, higher end-to-end WPT efficiencies can be achieved due to the improvement in antennas' gain [4], [9], [12]. Furthermore, the wide spectrum available enables the co-existence of several applications such as WPT and communications, enabling a massive mmWave-connected IoT [17]. However, the free space path loss and the additional NonLine-of-Sight (LoS) losses at mmWave bands, for example due to attenuation by water molecules at $28 \mathrm{GHz}$, may imply that WPT to wearables at mmWave bands is less efficient compared to UHF bands. In addition, the antennas need to maintain a broad bandwidth to benefit from the available spectrum. At an antenna-design level, existing flexible and wearable antenna implementations do not fully address both requirements, where the efficiency and bandwidth are improved at the expense of a thicker antenna [17].

mmWave-powered devices can be broadly classified into compact and large-area receivers. For a compact receiver, several analytical studies have shown that a higher antenna efficiency can be achieved at mmWave bands [10], [11]. Nevertheless, channel gain or s-parameter measurements have not been used to compare mmWave WPT to area-constrained devices. For large-area systems, beamforming rectennas [12], as well as large transmitting arrays have been investigated [9], [19], showing potential performance gains. However, the performance of a large-area mmWave rectenna array has not been compared, analytically or experimentally, to its similarlysized UHF counterpart. Therefore, this work aims to present a wearable antenna design suitable for mmWave WPT, as well as present an extensive evaluation of its performance as a compact and a large-area power receiver.

\section{A. mmWave Antenna \& System Requirements for WPT:}

To enable mmWave-powered WBANs, the following antenna design criteria need to be met: 
1) High-efficiency antennas with broad angular coverage for increased energy harvesting probability [20].

2) Conformable and low antenna profile for improved integration in clothing and on thinner substrates [21].

3) Broad bandwidth, to enable the antennas to be used in the full 5G spectrum [12], [17].

While several theoretical and practical studies have shown the benefits of mmWave WPT [4], [9], [12], the performance of a mmWave-powered network based on a real antenna, meeting the above criteria, remains unclear. To explain, the benefits of mmWave WPT compared to its UHF counterpart need to be evaluated. Therefore, a realistic investigation on the feasibility of mmWave WPT needs to account for:

1) Antenna radiation properties, based on real measured antenna radiation gain and patterns.

2) Realistic non-linear rectifier model, based on real commercially-available devices, to reflect different rectifiers' non-linear behavior.

3) Empirical LoS and N-LoS propagation models, to reflect the higher losses at mmWave bands.

\section{B. Summary of Contributions}

In this paper, a higher-order dual-mode broadband microstrip patch antenna is proposed on textiles for wearable WPT. The proposed antenna achieves:

1) The highest thickness-normalized efficiency of a wearable antenna.

2) Wider bandwidth compared to existing microstrip patch antennas.

3) The lowest profile of a broadside textile antenna with full body-isolation.

4) Wider angular coverage compared to wearable antennas with broadside off-body radiation.

The DC power output of a mmWave-powered WBAN based on the proposed antenna is then investigated numerically and experimentally demonstrating:

1) $11 \mathrm{~dB}$ measured basestation-to-body path (channel) gain improvement for an area-constrained receiver.

2) Up to $6.3 \times$ higher $\mathrm{DC}$ power output compared to a similar-sized UHF microstrip patch antenna in Line-ofSight $(\mathrm{LoS})$ and $2 \times$ higher power in Non-LoS.

3 ) the first analytical investigation of a mmWave-powered energy harvester based on a physical power conversion efficiency model

The proposed antenna design is introduced in Section II with the antenna measurements in Section III. The performance of the proposed antenna as a wireless receiver is then evaluated in Section IV, along with potential IoT applications of the proposed antenna, and mmWave WPT.

\section{Textile Patch Antenna Design and Simulation}

\section{A. Broadband Microstrip Antenna Design}

To design an antenna for wearable energy harvesting at mmWave bands the antenna needs to maintain a wide input bandwidth $\left(S_{11}\right)$. As mmWaves have very poor tissue penetration capabilities beyond the skin [22], the antenna's patterns
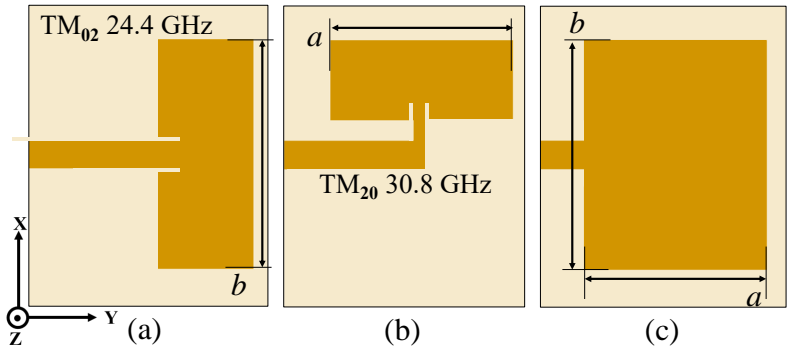

Fig. 1. Broadband antenna design steps: (a) $\mathrm{TM}_{20}$ patch at $f_{1}$; (b) $\mathrm{TM}_{02}$ patch at $f_{2} ;$ (c) dual-mode $\mathrm{TM}_{02 / 21}$ patch antenna.

need to be predominantly off-body. In addition, due to the wide variation in the angle of incidence of power, the antenna needs to cover a wide beamwidth. Previously, microstrip antennas with multiple radiative modes were used to obtain several resonances at nearby frequencies, improving the bandwidth compared to a conventional patch. To explain, the majority of reported flexible and wearable patch antennas resonate at a single-mode transverse magnetic (TM) mode, which is typically the first-order $\mathrm{TM}_{10}$ mode [23]. Nevertheless, to excite multiple modes simultaneously, a complex antenna structure is often needed [23], [24].

The dimensions of a rectangular microstrip patch antenna can be calculated analytically for a given TM resonant mode based on the length and width [25]. For a planar (i.e. $t<<\lambda$ ) microstrip cavity, the resonance frequency $f_{\mathrm{mn}}$ of a $\mathrm{TM}_{m n}$ mode is given by

$$
\begin{gathered}
f_{m n}=\frac{k_{m n} c}{2 \pi \sqrt{\epsilon_{r}}}, \\
k_{m n}=\sqrt{\left(\frac{m \pi}{a}\right)^{2}+\left(\frac{n \pi}{b}\right)^{2}},
\end{gathered}
$$

where $c$ is the speed of light, the resonant frequency $f_{m n}$ (1) and the wavenumber $k_{m n}$ (2) for the $m n$ mode can be calculated based on the dimensions [25]. $a$ and $b$ are the patch dimensions on the $m$ and $n$ respective axes.

To achieve a wide $S_{11}$ bandwidth covering the mmWave $5 \mathrm{G}$ bands $(24.25-29.50 \mathrm{GHz}$ [26]), the proposed antenna is designed to match $50 \Omega$ for two resonant TM modes. Firstly, a $\mathrm{TM}_{02}$ patch is designed with $b=9 \mathrm{~mm}$ as shown in Fig. 1-a. Given $\epsilon_{r}=1.95\left(1.9<\epsilon_{r}<2\right.$ due to the non-uniform gaps between the fabric and the polyimide), the $\mathrm{TM}_{02}$ patch has a calculated theoretical resonance at $24.3 \mathrm{GHz}$ using (1). Fig. 1-a shows the layout of the first $\mathrm{TM}_{02}$ patch iteration. The antenna is based on a $330 \mu$ m-thick woven-polyester fabric substrate, with a flexible $25 \mu \mathrm{m}$-thick polyimide interface layer. The total thickness of the substrate, including the polyimide and adhesive under the patch and the ground plane, has been measured using a micrometer to be $420 \mu \mathrm{m}$. The dielectric properties of the multi-layered substrate (polyimide+woven polyester+polyimide) have been measured using the phase delay and insertion losses in two microstrip lines, as detailed in [17], to be $\epsilon_{r}=1.95, \tan \delta=0.026$. The simulated $S_{11}$, obtained from CST Microwave Studio based on the substrate's measured properties, is shown in Fig. 2. 


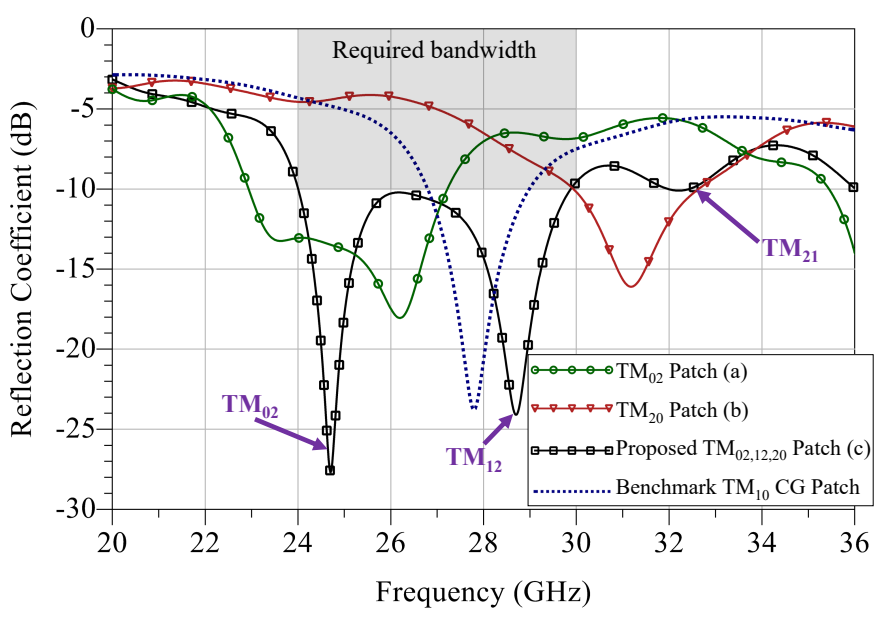

Fig. 2. Simulated $S_{11}$ of the single-mode patch cells and the proposed dualmode broadband antenna; a $\mathrm{TM}_{10} \mathrm{CG}$ patch is included for bench-marking.

The next step is designing a higher frequency patch. For $b=7.125 \mathrm{~mm}$, the analytical $\mathrm{TM}_{02}$ resonance, $f_{2}$, is $30.6 \mathrm{GHz}$. Fig. 1-b shows the layout of the $\mathrm{TM}_{02}$ patch. Then, a rectangular patch, Fig. 1-c, with the optimal dimensions $a=9.0$ and $b=7.13 \mathrm{~mm}$ is designed achieving $\mathrm{TM}_{02}, \mathrm{TM}_{12}$, and $\mathrm{TM}_{21}$ resonances at 24.3, 28.7, an $30.6 \mathrm{GHz}$. An inset microstrip feed with wide inset slots, as shown in Fig. 3-a, matching the impedance of the antenna at the $\mathrm{TM}_{02}$ and $\mathrm{TM}_{12}$ resonances to achieve an $S_{11}$ under $-10 \mathrm{~dB}$, as shown in Fig. 2 .

For benchmarking, a common geometry (CG) $\mathrm{TM}_{10}$ patch with a $\lambda / 4$ impedance transformer has been simulated, showing a narrower bandwidth in Fig. 1 than the individual patch cells resonating at the second TM mode, and a significantly narrower bandwidth than the proposed antenna. As the $S_{11}$ is dependent on the substrate, the antenna's bandwidth may change when implemented on a low-loss substrate. The antenna was simulated on a low-loss substrate $(\tan \delta=0.0007)$. On such a substrate, the proposed antenna maintains a $S_{11}<-6 \mathrm{~dB}$ (Voltage Standing Wave Ratio $<3$ ) from 22.5 to $26.7 \mathrm{GHz}$ instead of $\operatorname{VSWR}<2$, while this may restrict the antenna's use in applications where a power amplifier-antenna VSWR is under 2.0, it does not affect the antenna's applicability to WPT, where a VSWR exceeding 2 does not risk damaging other system components.

The electric (E-field) distribution of the individual singlemode $\mathrm{TM}_{20} / \mathrm{TM}_{02}$ patches are shown in Fig. 4-a and 4$\mathrm{b}$, respectively. By observing the E-field, both antennas are expected to have broadside far-field patterns with a wide $-3 \mathrm{~dB}$ beamwidth. From CST, the single patch cells (Fig. 1 -a and b) achieve a $95^{\circ}$ and $102^{\circ}$ half-power beamwidth of at 24.5 and $30.5 \mathrm{GHz}$, respectively. The peak simulated directivity is 8.5 and $7.5 \mathrm{dBi}$ at 24.5 and $30.5 \mathrm{GHz}$. The proposed patch in Fig. 3-a achieves a simulated broadside peak directivity of $10 \mathrm{dBi}$, owing to its larger physical aperture compared to a standard $\mathrm{TM}_{10}$ patch. Only the directivity has been simulated at this stage as a loss-less dielectric and conductor were considered.

For the measurement prototype, a prolonged microstrip feed was then introduced to minimize any distortion from the con-

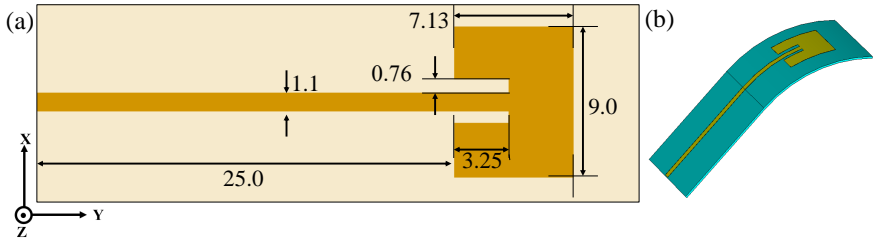

Fig. 3. The proposed dual-mode microstrip antenna: (a) layout and dimensions (in $\mathrm{mm}$ ) of the fabricated prototype, (b) simulation model of the bent antenna.

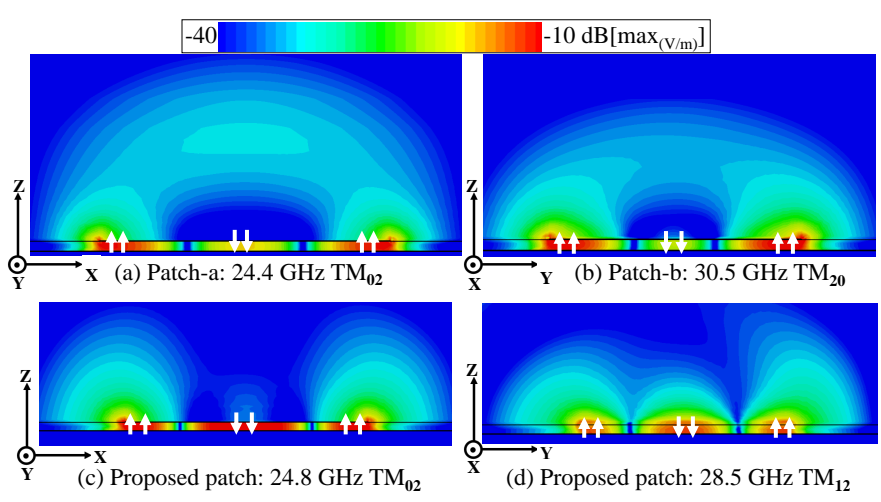

Fig. 4. Simulated $E$-fields across the antenna: (a) single-cell $\mathrm{TM}_{20}$ patch; (b) single-cell $\mathrm{TM}_{02}$ patch; (c) proposed patch $\mathrm{TM}_{20}$ mode at $24.8 \mathrm{GHz}$; (d) proposed patch $\mathrm{TM}_{12}$ mode at $28.5 \mathrm{GHz}$.

nector, due to additional reflections, to the antenna's radiation properties [5]. The additional losses in the microstrip feed will be representative of the insertion losses in a distributed microstrip matching network, should the antenna be matched to a rectifier, or in an array feed network.

In addition to the improved bandwidth due to matching the antenna at two different modes, operating the antenna at a higher-order mode improves its angular coverage in the broadside off-body direction. To explain, a typical textilebased TM10 patch antenna has a half-power beamwidth $\approx 60^{\circ}$ in the off-body direction [27]. It was shown previously that a wider angular coverage correlates to a higher energy harvesting probability and, on-average, higher DC power [20]. A second-order patch on the other hand exhibits a broader beam, as shown in Fig. 5-b.

As observed in Fig. 5, the proposed antenna achieves two key advantages over a standard $\mathrm{TM}_{10} \mathrm{CG}$ patch:

1) Achieving wider bandwidth, both for a single resonant mode (Fig. 2), and for dual-mode operation (Fig. 5-a);

2) Maintaining wider angular coverage, as shown in Fig. 5$\mathrm{b}$, where the $-10 \mathrm{~dB}$ beamwidth of the proposed antenna is $50^{\circ}(38 \%)$ wider than that of a CG patch.

To explain, in spite of the observed bore-sight "null" of the $\mathrm{TM}_{20}$ mode, the broadside angular coverage of the proposed antenna will result in better energy harvesting coverage from arbitrarily-directed radiation, which was previously found to improve the DC power harvested compared to narrower-beam higher-gain antennas [20].

\section{B. Bending and Wearable Operation}

As the antenna is designed for wearable applications, it is essential to simulate the antenna in proximity with a human 


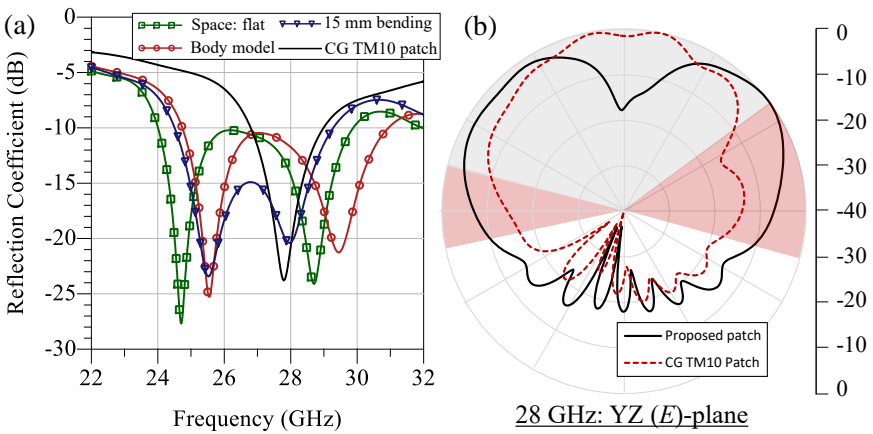

Fig. 5. Comparison of the proposed patch with a common geometry (CG) $\mathrm{TM}_{10}$ patch: (a) bandwidth; (b) radiation pattern, where the shaded red area indicates the angular coverage improvement.

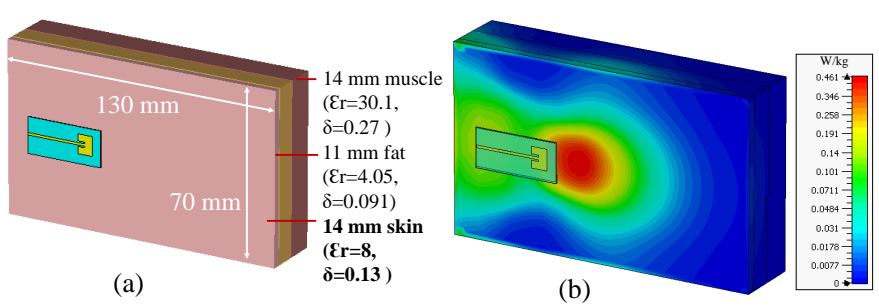

Fig. 6. Simulation of the patch antenna on the body model: (a) body phantom structure, (b) simulated SAR at $28 \mathrm{GHz}$ for a $0.5 \mathrm{~W}$ input.

tissue model. Due to the short wavelength and to simplify the electromagnetic problem reducing the solver's time, a simplified layered tissue model is used. The skin layer, shown in Fig. 6, is based on the measured skin properties above $26.5 \mathrm{GHz}$, reported in [22]. It was previously shown that mmWaves do not penetrate deeper than the skin layer [28]. Fig. 6-a shows the model of the patch operating at $3 \mathrm{~mm}$ separation from the human model, as well as the dielectric properties and thickness of each layer. The simulated reflection coefficient on the tissue model shows less than $2 \%$ shift in the antenna's resonance when placed on the body, this is attributed to the additional capacitance introduced by the higher $\epsilon_{r}$ of the tissue compared to air. The impact of bending has been investigated by bending the antenna across a $15 \mathrm{~mm}$ radius, as shown in Fig. 3-b, which results in less than $4 \%$ shift in the resonance, as shown in Fig. 5, and does not alter the $\mathrm{S}_{11}<-10 \mathrm{~dB}$ bandwidth from 25 to $28.5 \mathrm{GHz}$.

The tissue model has been used to calculate the antenna's Specific Absorption Rate (SAR) at $28 \mathrm{GHz}$, shown in Fig. 6$\mathrm{b}$, to showcase the antenna's compliance with the IEEE C95.1 SAR limits. The highest absorption is observed in the skin layer, as most of the mmWave power does not penetrate deeper than the skin layer [28]. The maximum SAR of $0.461 \mathrm{~W} / \mathrm{kg}$, when normalized to $10 \mathrm{~g}$ tissue mass, is compliant with the IEEE C95.1 regulatory limit of $1.6 \mathrm{~W} / \mathrm{kg}$. The SAR was calculated for a $0.5 \mathrm{~W}$ input, which is higher than the maximum power level a textile mmWave rectenna may be exposed to up to $1 \mathrm{~cm}$ from the transmitter [17].

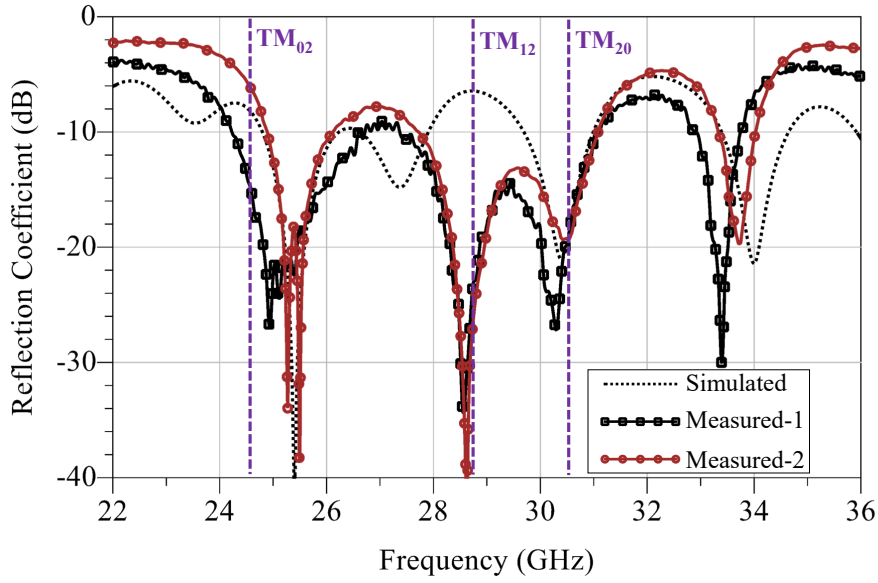

Fig. 7. Measured reflection coefficient of two antenna prototypes (solid) and the CST simulation with a soldered connector (dashed).

\section{Antenna MeAsurements}

\section{A. Antenna Bandwidth}

The antenna has been fabricated using photolithography on flexible polyimide copper laminates, using the method detailed in [29], which was previously used to realize antennas resilient to bending [21] and machine washing [29]. Utilizing thin and flexible copper laminates has been widely reported to be more suitable for mmWave antennas compared to conductive fabrics, owing to the ability to resolve smaller features [5], [17]. The patch antenna trace is adhered to the top of the fabric with the continuous ground realized using the same copper laminates, attached to the bottom layer of the substrate.

An Agilent E8361A PNA 67 GHz Vector Network Analyzer (VNA) calibrated using a standard e-calibration kit has been used to measure the antenna's s-parameter's response. Two antenna prototypes have been used to measure the antenna's reflection coefficient. By using two prototypes, the uncertainty introduced by the connector's soldered interface can be observed and minimized. Due to the thin textile substrate compared to conventional circuit boards, the $1.6 \mathrm{~mm}$-thick connector and pin are expected to introduce additional discontinuities at the coax/microstrip interface, altering the $S_{11}$ response. The connector-fed antenna has been simulated in CST. Fig. 7 shows the simulated and measured reflection coefficient of the connector-fed antenna, along with the analytically calculated resonant TM modes using (1).

From Fig. 7, the simulated (with the connector) and measured antenna's reflection coefficient demonstrate an $S_{11}<$ $-10 \mathrm{~dB}$ bandwidth between 24.9 to $31.1 \mathrm{GHz}$. The analytically calculated resonant frequencies of each mode agree with the measured resonances of both prototypes. From 26 to $28 \mathrm{GHz}$, a discrepancy can be observed between the two prototypes. This can be attributed to the length of the connector's unshielded pin, approximately $\lambda / 4$ at $27 \mathrm{GHz}$, which can act as an impedance transformer around $27 \mathrm{GHz}$ causing additional reflection [30], as observed in the $S_{11}$ of prototype- 2 in Fig. 7. For both measured prototypes, the antenna maintains an impedance bandwidth between 24.9 and 


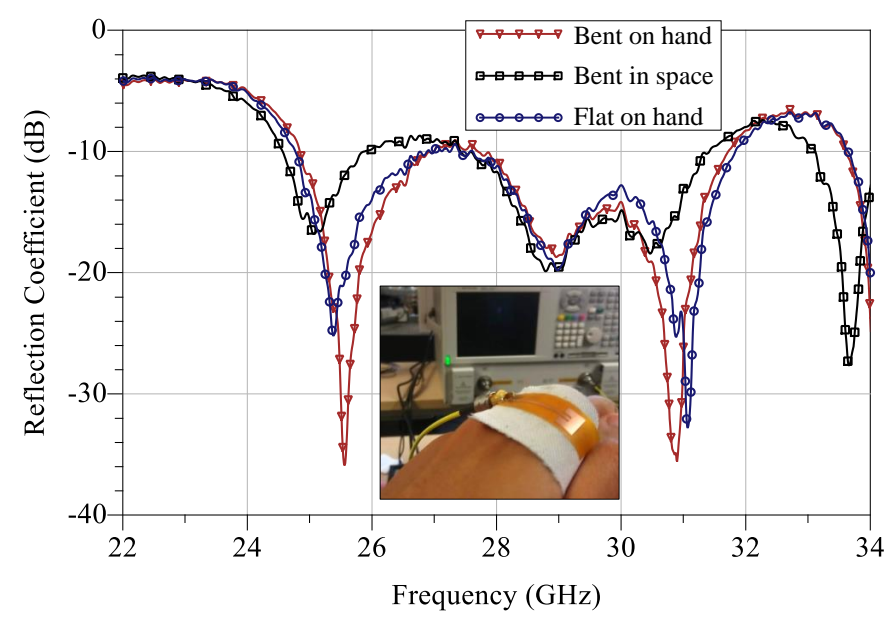

Fig. 8. Measured reflection coefficient of the microstrip antenna under bending and near the body; inset shows the on-hand bending.

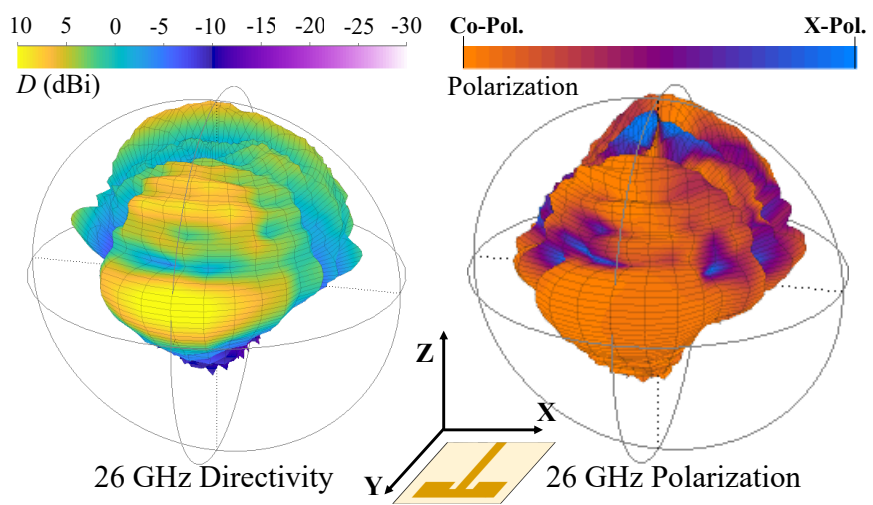

Fig. 9. Measured 3D directivity $D$ and polarization of the proposed antenna, on a large ground plane, at $26 \mathrm{GHz}$.

$31.1 \mathrm{GHz}$, with an $S_{11}$ under -8 and $-10 \mathrm{~dB}$ on prototype 1 and 2 , respectively.

In wearable operation, the antenna needs to withstand bending and maintain its bandwidth in human proximity. The antenna's $S_{11}$ was measured on-body and under bending. The antenna was bent both in space and on-body, as shown in the inset in Fig. 8, while measuring the $S_{11}$. As observed in Fig. 8, the antenna's $S_{11}$ response remains mostly unaffected in human proximity and under bending.

\section{B. Antenna Radiation Patterns}

The 3D polarimetric radiation patterns of the proposed antenna were measured in an anechoic chamber at 24 and $26 \mathrm{GHz}$; the measurements were limited by the available power amplifier's operation frequency and the WR-42 horn cut-off frequency. To improve the repeatability of the measurements, the antenna was mounted on a large (radius $>5 \lambda_{0}$ ) circular ground plane, as in [17]. Fig. 9 shows the 3D measured directivity $D$, normalized to $10 \mathrm{dBi}$, and the polarization of the measured $D$. The polarization of the textile antenna has been normalized using a standard $20 \mathrm{dBi}$ WR- 42 horn.

The peak measured $D$ is 13.2 and $11.2 \mathrm{dBi}$, at 24 and $26 \mathrm{GHz}$ respectively. The measured results are indicative of the antenna's performance over a continuous ground plane, such as a fully-textile shielding between the antenna and the human body, which was shown to reduce the SAR of mmWave wearable antennas [17]. The simulated and measured relative gain patterns of the antenna, with the connector and the large ground plane, are shown in Fig. 10. The underlined plane in Fig. 10 indicates the principal plane of the respective TM mode at each frequency. It can be observed that the simulated and measured co-polarized $D$ agree in the main off-body forward beam direction $\left(-90^{\circ}<\theta<90^{\circ}\right)$, and that the antenna's main lobe on the Z-axis is broader than a standard patch (Fig. 5-b), enabling more position-tolerant energy harvesting [20], [31].

On the principal plane, the antenna achieves 13 and $25 \mathrm{~dB}$ co/cross-polarization at 24 and $26 \mathrm{GHz}$ respectively. Polarization purity is often regarded as a figure of merit in antenna design for communications. However, in the context of energy harvesting, a high co/cross-polarization isolation does not translate to better energy harvesting performance. It was previously shown that both directivity and polarization can almost be irrelevant when the receiver is far from the basestation, and that dual-polarization using orthogonal antennas in a large-area implementation are needed for polarizationinsensitive harvesting [32]. On the other hand, while circular polarization can be preferred in directional WPT [33], it is still prone to mismatch between right and left-hand circularly polarized incident radiation [34]. Finally, in the more general wearable WBAN antenna case, a high polarization purity is not required due to the mobility and rotation of the antenna, for example when the antenna is used on the wrist or arm [35].

As the antenna utilizes two orthogonal modes, the polarization changes across the $S_{11}$ bandwidth. In WPT applications, utilizing two orthogonally-positioned antennas with power combining can mitigate the polarization mismatch between the transmitter and the textile-based power harvesting antenna [36]. By using two harvesting elements with hybrid coupling, the antenna will be able to harvesting the incident linearlypolarized radiation across its full bandwidth.

The 3D total radiated power was used to measure the efficiency (inclusive of mismatch) of the proposed antenna, with respect to a reference antenna. A WR-42 horn antenna was used as a reference using the method detailed in [37]. The radiated power of the textile antenna and the reference horn were measured at 24 and $26 \mathrm{GHz}$. Three measurements of both the antenna-under-test and the reference horn were performed to minimize the uncertainty; a $1.3 \%$ standard deviation was observed. The measured $S_{11}$ of the connectorized antenna was used to calculate the radiation efficiency excluding mismatch. The antenna's measured radiation efficiency is $60 \%$ and $59 \%$, $\pm 5 \%$ (based on the technique in [37]), at 24 and $26 \mathrm{GHz}$ respectively. The simulated efficiency is 55\% and 53\%, at 24 and $26 \mathrm{GHz}$, agreeing within 5\% with the measured efficiency. Using the measured efficiency, the antenna's measured gain is $8.2 \mathrm{dBi}$ at $26 \mathrm{GHz}$.

Table I compares the proposed textile-based antenna design to reported mmWave microstrip antennas. It can be seen that the proposed antenna achieves the widest fractional bandwidth of $22 \%$ compared to other single-layer antennas, owing to 


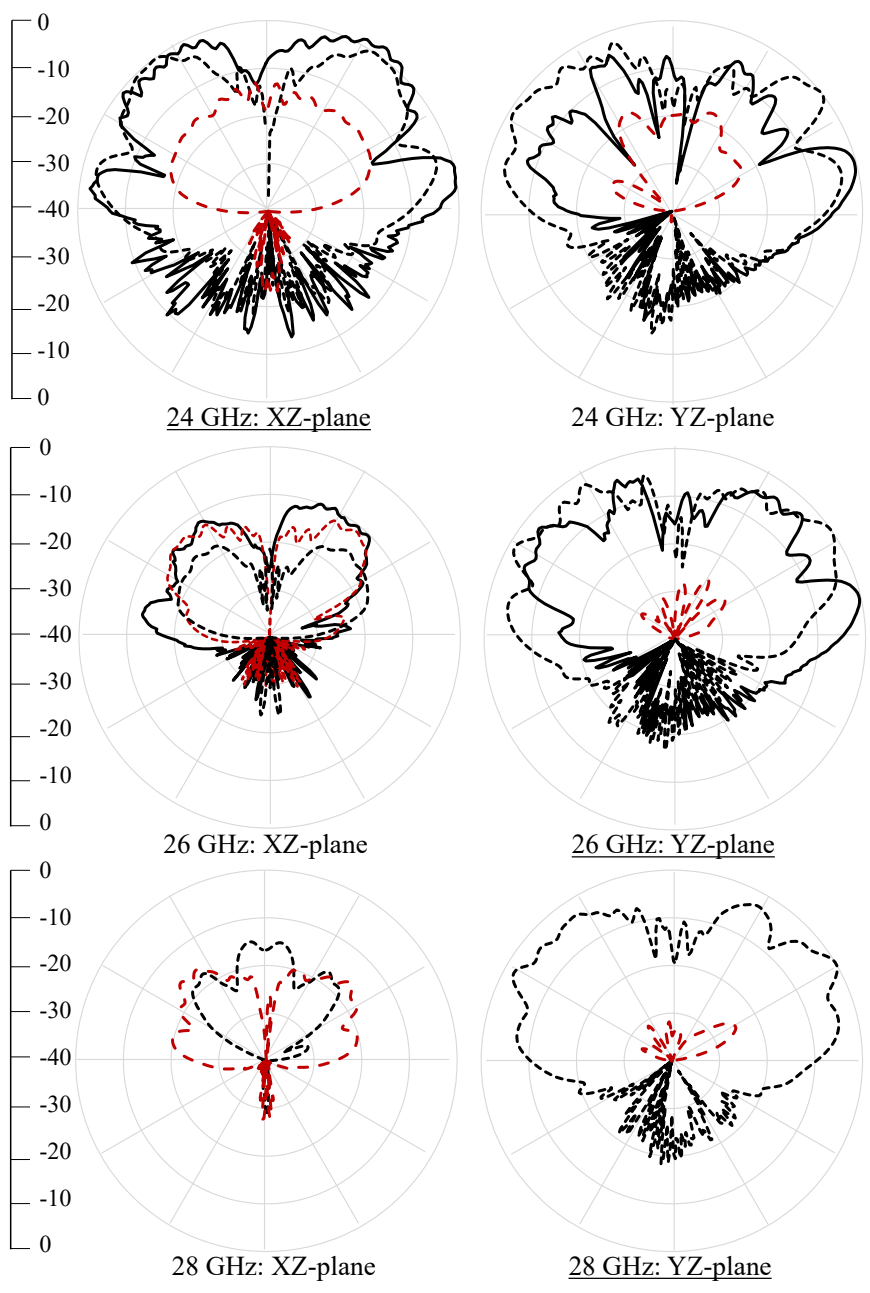

Fig. 10. Measured (solid) and simulated (dashed) co-polarized (black) and cross-polarized (red) relative gain (normalized) radiation patterns of the proposed antenna.

its dual-resonant structures. To compare the efficiency with reported mmWave antennas, the measured efficiency was normalized to the substrate's electrical thickness using

$$
\eta_{\text {norm. }}=\eta_{\text {rad. }} \times \frac{\lambda_{0}}{t},
$$

where $t$ is the antenna's thickness, $\lambda_{0}$ is the wavelength at the lower bound of the antenna's $S_{11}$ bandwidth, and $\eta_{\text {rad. }}$ is the measured antenna efficiency. This metric allows the evaluation of the antenna's efficiency with respect to its volume, whereas the aperture efficiency only considers the area [38].

Compared to the textile antennas in [27] and [5], operating at $60 \mathrm{GHz}$, the proposed antenna achieves a higher radiation efficiency while having a lower profile (compared to $\lambda_{0}$ ). While [17] achieves a higher efficiency using a reflectorbacked textile antipodal antenna, which increases the thickness and complexity of the antenna. However, the proposed antenna compares favorably when $\eta_{\text {norm. }}$ is considered, as visualized in Fig. 11.

Compared to the multi-mode patch in [24], the proposed antenna compares favorably when the antenna efficiency is normalized to the thickness, despite being implemented on

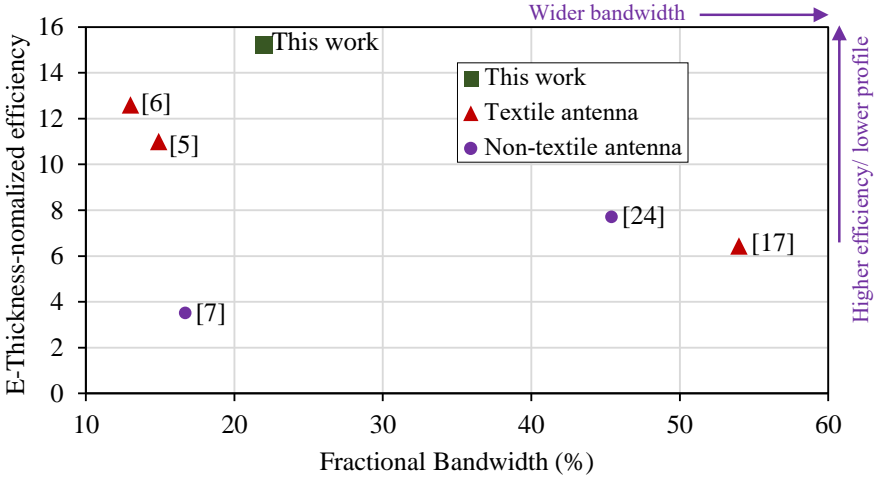

Fig. 11. Comparison of recent microstrip antennas based on the thicknessnormalized efficiency $\eta_{\text {norm. }}$, and the fractional bandwidth.

textiles and not RF laminates. In addition, the wider bandwidth in [24] was achieved using a Substrate-Integrated Waveguide (SIW) cavity and a very thick $\left(0.11 \lambda_{0}\right)$ substrate. While broadband "wire-type" antennas such as [39] achieve higher efficiency and bandwidth on low-loss substrates, the antenna requires double-sided etching and the efficiency is only achievable with $\tan \delta<0.001$ substrates. Other printed $\mathrm{TM}_{10}$ patches, [40], [41], implemented on low-cost substrates or using printed conductors achieve a narrower bandwidth and a lower radiation efficiency than the proposed antenna.

\section{MmWaVe Wireless-Powered BAN Evaluation}

In this section, the power harvesting performance of two mmWave-powered applications based on the proposed antenna is evaluated. We first compare the WPT efficiency based on the proposed patch to a similar sized off-body antenna based on the same area, in Section IV-A, demonstrating that a higher-efficiency WPT link can be achieved at mmWave bands compared to $2.4 \mathrm{GHz}$, when the antennas' area is restricted. In section IV-B, the wireless power harvesting capabilities of a large-area rectenna system based on the proposed antenna is evaluated and compared to a sub- $1 \mathrm{GHz}$ UHF off-body rectenna.

\section{A. Compact Single-Receiver}

Higher power can be delivered to a compact receiver in mmWave bands due to the comparable physical aperture size of the antenna to the wavelength. The improved antenna:wavelength ratio for area-constrained devices has previously motivated miniaturized RF-powered devices to be designed for frequencies above $20 \mathrm{GHz}$ [11]. In [4], it was analytically shown that for antennas of a fixed area, the power received increases with the frequency. This section experimentally demonstrates the benefits of using the proposed antenna for LoS WPT for a transmitter and receiver with areaconstrained antennas.

Two symmetric textile patch antennas were connected to a VNA's ports to measure the forward transmission between them in space, as shown in Fig. 12. As the proposed antenna is only matched for mmWave bands and will have a very high reflection coefficient for UHF bands, the measured forward 
TABLE I

COMPARISON OF THE PROPOSED TEXTILE ANTENNA WITH REPORTED PLANAR MMWAVE MICROSTRIP ANTENNAS.

\begin{tabular}{|c|c|c|c|c|c|c|c|c|c|}
\hline Study & Antenna & Substrate & $\begin{array}{l}\text { Bandwidth } \\
\text { (GHz) }\end{array}$ & $\begin{array}{l}\text { Fractional } \\
\text { BW }\end{array}$ & $\begin{array}{l}\text { Antenna } \\
\text { efficiency }\end{array}$ & $\eta_{\text {norm. }}$. & Gain (dBi) & $\begin{array}{l}\text { Co/x-pol. } \\
\text { isolation } \\
\text { (dB) }\end{array}$ & $\begin{array}{l}\text { Electrical } \\
\text { volume** } \\
\left(\lambda_{0}^{3}\right)\end{array}$ \\
\hline This work & $\begin{array}{l}\mathbf{T M}_{02} / \mathbf{T M}_{12} \\
\text { Patch }\end{array}$ & $\begin{array}{l}\text { Kapton } \\
\text { on woven } \\
\text { polyester } \\
(\tan \delta=0.026) \\
\end{array}$ & $24.9-31.1$ & $22 \%$ & $\begin{array}{l}\mathbf{6 0 \%} \\
( \pm 5 \%)\end{array}$ & 15.2 & $\begin{array}{l}7.7 \text { at } 24 \\
\text { GHz; 8.9 at } \\
26 \mathrm{GHz}\end{array}$ & $\begin{array}{ll}13 \text { at } & 24 \\
\mathbf{G H z}^{*} ; & \\
25 \text { at } & 26 \\
\mathbf{G H z}^{*} & \\
\end{array}$ & $\begin{array}{l}0.59 \times \\
0.75 \times \\
0.034\end{array}$ \\
\hline $\begin{array}{l}\text { AWPL'12 } \\
\text { [6] }\end{array}$ & $\begin{array}{l}\text { End-fire } \\
\text { quasi-Yagi }\end{array}$ & $\begin{array}{l}\text { Woven } \\
\operatorname{cotton} \\
(\tan \delta=0.016)\end{array}$ & $57-65$ & $13 \%$ & $48 \%$ & 12.6 & 11.9 & 13 & $\begin{array}{ll}3 \times & 0.76 \times \\
0.04 & \end{array}$ \\
\hline TAP'13 [5] & $\begin{array}{l}2 \times 2 \mathrm{TM}_{10} \\
\text { patch array }\end{array}$ & $\begin{array}{l}\text { Woven } \\
\operatorname{cotton} \\
(\tan \delta=0.02)\end{array}$ & $56-65$ & $14.9 \%$ & $41 \%$ & 11.0 & 9 & $\begin{array}{l}15 \text { (meas.); } \\
>20 \text { (sim.) }\end{array}$ & $\begin{array}{l}1.10 \times 0.96 \\
\times 0.037\end{array}$ \\
\hline $\begin{array}{l}\text { TAP'19 } \\
\text { [24] }\end{array}$ & $\begin{array}{l}\mathrm{TM}_{10} / \mathrm{TM}_{10} \\
\text { slotted } \\
\text { patch }\end{array}$ & $\begin{array}{l}\text { Taconic } \\
+\quad \text { Rogers } \\
(\tan \delta<0.001)\end{array}$ & $30-50$ & $45.4 \%$ & $85 \% *$ & 7.7 & $5-8.5$ & $\begin{array}{l}16 \text { (meas.); } \\
25 \text { (sim.) }\end{array}$ & $\begin{array}{l}1.25 \times \\
1.25 \times \\
0.110 \\
\end{array}$ \\
\hline $\begin{array}{l}\text { AWPL'19 } \\
\text { [39] }\end{array}$ & $\begin{array}{l}\text { Archimedean } \\
\text { spiral }\end{array}$ & $\begin{array}{l}\text { Duroid } \\
5880 \\
(\tan \delta<0.001)\end{array}$ & $21.1-34.1$ & $46.4 \%$ & $96 \% *$ & 53.7 & 6.5 & $\approx 7^{\dagger}$ & $\begin{array}{l}1.06 \times 1.06 \\
\times 0.013\end{array}$ \\
\hline $\begin{array}{l}\text { TMTT'20 } \\
\text { [17] }\end{array}$ & $\begin{array}{l}\text { Reflector- } \\
\text { backed } \\
\text { antipodal } \\
\text { Vivaldi }\end{array}$ & $\begin{array}{l}\text { Kapton- } \\
\text { on-woven } \\
\text { polyester, } \\
\tan \delta=0.02\end{array}$ & $24-40 \mathrm{GHz}$ & $54 \%$ & $67 \%$ & 6.44 & 8 & NR & $\begin{array}{c}0.33 \times 0.33 \\
\times 0.00027\end{array}$ \\
\hline
\end{tabular}

NR: not reported; ${ }^{*}$ Simulated result; ${ }^{\dagger}$ right/left-hand circular polarization isolation (estimated from the patterns); ${ }^{* *}$ volume normalized to the minimum $S_{11}<-10 \mathrm{~dB}$ frequency.

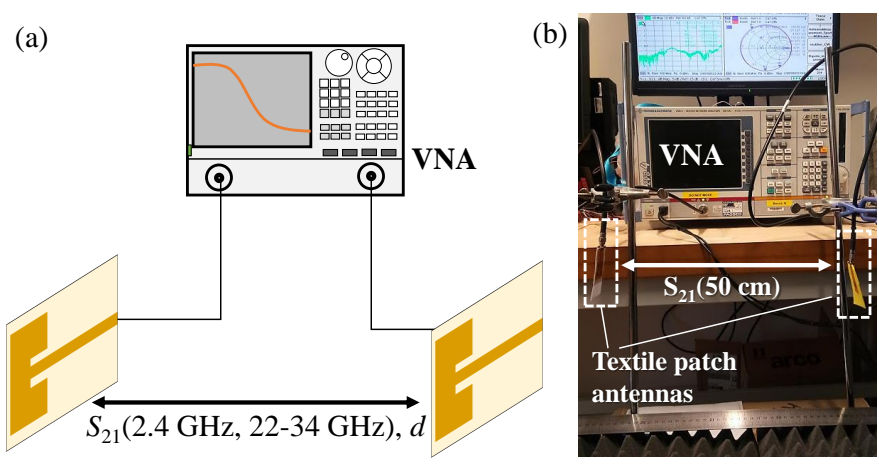

Fig. 12. Path gain measurements between two symmetric $7.13 \times 9 \times 0.5 \mathrm{~mm}^{3}$ antennas at $50 \mathrm{~cm}$ at UHF and mmWave: (a) schematic; (b) photograph of the UHF setup.

transmission has been corrected post-measurement to exclude the impedance mismatch losses at $2.4 \mathrm{GHz}$. The mismatch losses have been excluded using the measured impedance based on the $S_{11}$ of the antenna at $2.4 \mathrm{GHz}$, and re-calculating the $S_{21}$ based on $Z_{\text {source }}=Z_{\text {antenna }}^{*}$. By positioning the antennas at $d=50 \mathrm{~cm}, d$ is equal to $4 \times \lambda_{2.4 \mathrm{GHz}}$ ensuring the antennas are in the far-field.

Fig. 13 shows the measured forward $S_{21}$ from 0.5 to $4 \mathrm{GHz}$ and from 22 to $34 \mathrm{GHz}$. It can be seen that due to the antenna's improved gain and efficiency in mmWave bands, it is possible to achieve over $11 \mathrm{~dB}$ higher $S_{21}$ at $26.5 \mathrm{GHz}$ compared to $2.4 \mathrm{GHz}$, despite the increased path loss, even after excluding the mismatch losses at $2.4 \mathrm{GHz}$. The additional advantage of using patch antennas for WPT in mmWave bands is the ability to implement the off-body patch on a lowprofile substrate, as shown in Fig. 11. Moreover, while the impedance mismatch losses were excluded at $2.4 \mathrm{GHz}$ for a

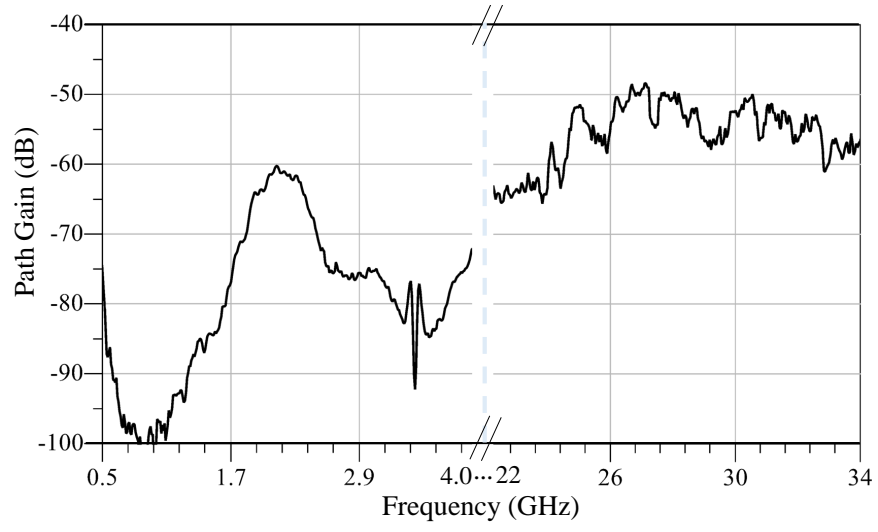

Fig. 13. Measured path gain $\left(S_{21}\right)$ between two symmetric antennas at 50 $\mathrm{cm}$ at $\mathrm{UHF}$ and mmWave.

fair comparison, achieving a low $S_{11}$ at $2.4 \mathrm{GHz}$ with such a compact antenna $\left(0.06 \times 0.07 \lambda_{2.4 \mathrm{GHz}}^{2}\right)$ will require a matching network, adding to the insertion losses and the overall cost and complexity of the WPT solution.

\section{B. Large-Area Implementation}

Where area is not a constraint, a large-area implementation can be used [16]. An array of rectennas based on the proposed antenna is considered. Each rectenna is formed of a single element rectifier. As the power is combined at DC, the large area rectenna does not result in a more directional radiation pattern, and hence does not reduce the antennas' harvesting beamwidth. DC combining has previously been demonstrated with only $1 \%$ loss [36], based on two linearly-polarized patch antennas and a hybrid coupler. In our analysis, loss-less DC combining is assumed. Fig. 14-a shows the architecture of 


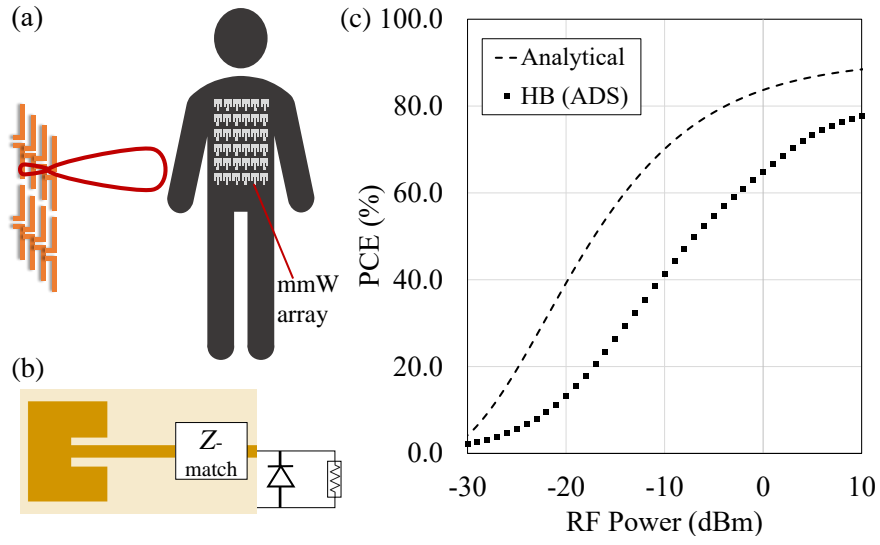

Fig. 14. The large-area mmWave power receiver: (a) textile-based rectenna array with directional transmitter and ideal DC combining, (b) a single rectenna cell, (c) analytically calculated and simulated PCE of the rectifier at $28 \mathrm{GHz}$.

the large-area mmWave rectenna array, and the rectenna's structure in Fig. 14-b.

In previous evaluations of mmWave energy harvesting networks, an ideal Power Conversion Efficiency (PCE) was used [9]. Both the linear [9] and non-linear models [19] previously used were not based on actual rectifier devices. In addition, a quadratic PCE formula may not accurately model all the physical loss contributors such as diode losses and harmonic generation [42]. The analytical diode PCE formulas proposed in [42] are used as a starting point for the PCE evaluation. However, as discussed in [43], such analytical closed form approximations do not accurately reflect the losses due to harmonics generation at very low power levels, and Harmonic Balance (HB) simulation is more suited. Therefore, in our comparison between mmWave and UHF WPT performance, $\mathrm{HB}$ results are used for a more conservative estimate.

The diode considered in this work is the VDI W-band ZBD GaAs Schottky diode, used for low-power rectification up to $100 \mathrm{GHz}$ for its low forward voltage and parasitic capacitance [44]. Fig. 14-c shows the calculated PCE for the rectifier using the analytical formulas from [42] and HB simulation. The peak PCE of $77 \%$ observed in the HB model is within $10 \%$ of the reported PCE at $36 \mathrm{GHz}$ in [45], based on commercial Schottky diodes [4].

On the other hand, commercial Schottky diodes implemented in specialized low-yield GaAs processes such as the VDI diode may be regarded as high-cost components unsuitable for scalable IoT applications [4]. Nevertheless, recently reported rectifiers operating beyond $60 \mathrm{GHz}$ have been fabricated in low-cost mass production-friendly standard CMOS processes. Therefore, future implementations of large-area mmWave-powered systems could be realized using low-cost rectifiers integrated with digital circuitry in a CMOS IC [46], improving the integration of the overall system and reducing its complexity. CMOS mmWave rectifiers have been reported with sub-0 dBm high-efficiency harvesting capabilities which indicates that their performance is comparable to Schottkybased systems. For example, a $1 \mathrm{~V}$ DC output from $-13 \mathrm{dBm}$ was reported at $61 \mathrm{GHz}$ [47], with a 9\% PCE, showing the potential for low-cost and high-efficiency rectification in the $5 \mathrm{G} \mathrm{K} / \mathrm{Ka}$ bands.

Two transmitters are considered: a $28 \mathrm{GHz} 53 \mathrm{dBm}$ Equivalent Isotropically Radiated Power (EIRP) and an $892 \mathrm{MHz}$ (the center resonant frequency of [48]) $36 \mathrm{dBm}$ EIRP. These are the maximum permissible EIRP levels for both bands. In mmWave bands, the maximum EIRP is higher than sub-5 GHz bands. For example, an EIRP of $75 \mathrm{dBm}$ is permitted for a $5 \mathrm{G}$ basestation, where the higher EIRP is achieved using high-gain phased arrays [49]. The $53 \mathrm{dBm}$ EIRP could be realized using a $1 \mathrm{~W}$ transmitter and a $23 \mathrm{dBi}$ antenna, which is permissible for license-free bands above $5 \mathrm{GHz}$ [50]. Below $5 \mathrm{GHz}$, the EIRP limit is capped at $4 \mathrm{~W}$ (approximately $36 \mathrm{dBm}$ ). From a practical implementation perspective, the antenna's theoretical minimum physical aperture area $A_{\text {Phys. }}$ can be calculated from the aperture efficiency $e_{a}$ using

$$
A_{\text {Phys. }}=\frac{G \lambda^{2}}{4 \pi e_{a}}
$$

for a fixed $e_{a}$ and a target antenna gain $G$ (dimensionless) [51]. Assuming a $90 \%$ aperture efficiency, typical for radiating aperture antennas [51], a $23 \mathrm{~dB}$ antenna at $28 \mathrm{GHz}$ would occupy an area of $2000 \mathrm{~mm}^{2}$. This represents $95 \%$ less area than a $6 \mathrm{dBi}$ antenna operating at $900 \mathrm{MHz}$. Therefore, utilizing mmWave wireless charging base-stations promises reduced base-station antenna size allowing more pervasive deployment in future IoT micro-cells.

Multiple array sizes based on the proposed antenna are considered, with a $5.4 \mathrm{~mm}(\lambda / 2$ at $28 \mathrm{GHz})$ spacing between the array elements. The power harvested by the rectennas is calculated using an empirical propagation model. The UHF textile patch antenna presented in [48] for sub-1 GHz WPT is considered as a reference to evaluate the performance of mmWave powering of WBANs. For both mmWave and UHF, the maximum PCE obtained from the HB simulation is used.

Fig. 15 shows the received power by the UHF and mmWave antennas. Although free-space propagation losses at $28 \mathrm{GHz}$ are significantly higher compared to $892 \mathrm{MHz}$, the increased array size allows more power to be recovered compared to the UHF patch, for arrays of $6 \times 6$ elements or larger.

The area consumed by the UHF patch is $190 \times 240 \mathrm{~mm}^{2}$ [48], considering a single mmWave array element size of $9 \times 14 \mathrm{~mm}^{2}$ and a $5.4 \mathrm{~mm}$ separation between the elements, a $13 \times 13$ array could be realized in approximately the same area as the UHF patch. The $13 \times 13 \mathrm{mmWave}$ array is considered for further evaluation of the $28 \mathrm{GHz}$-powered WBAN case. To provide more accurate modeling of the propagation losses, an empirical path loss exponent is used to calculate the power received by the antennas at $28 \mathrm{GHz}$. The received DC power $P_{\mathrm{RX}}$ has been calculated using

$$
P_{\mathrm{RX}}=P_{\mathrm{EIRP}} G_{R X}\left(\frac{c}{4 \pi \lambda}\right)^{2} \frac{1}{d^{\alpha}} \times \mathrm{PCE},
$$

where $\alpha$ is the path loss exponent for the distance $d$, and the PCE is the HB-simulated PCE shown in Fig. 14-c. $\alpha=$ 2.0 and $\alpha=2.9$, for LoS and N-LoS respectively, based on the empirical channel models of $28 \mathrm{GHz}$ propagation in [52]. Only free-space LoS propagation is considered for the UHF 

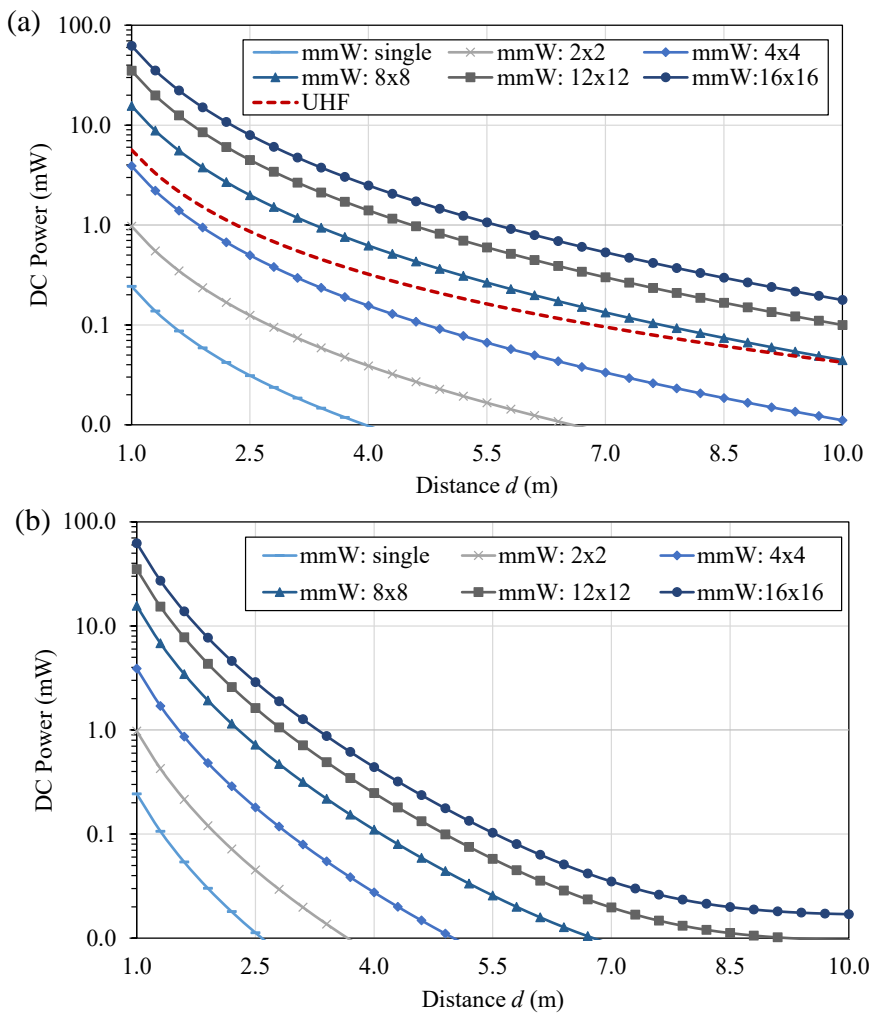

Fig. 15. DC power harvested by different-sized arrays, based on the measured antenna gain and empirical path loss model, at $28 \mathrm{GHz}$ (a) LoS, (b) N-LoS.

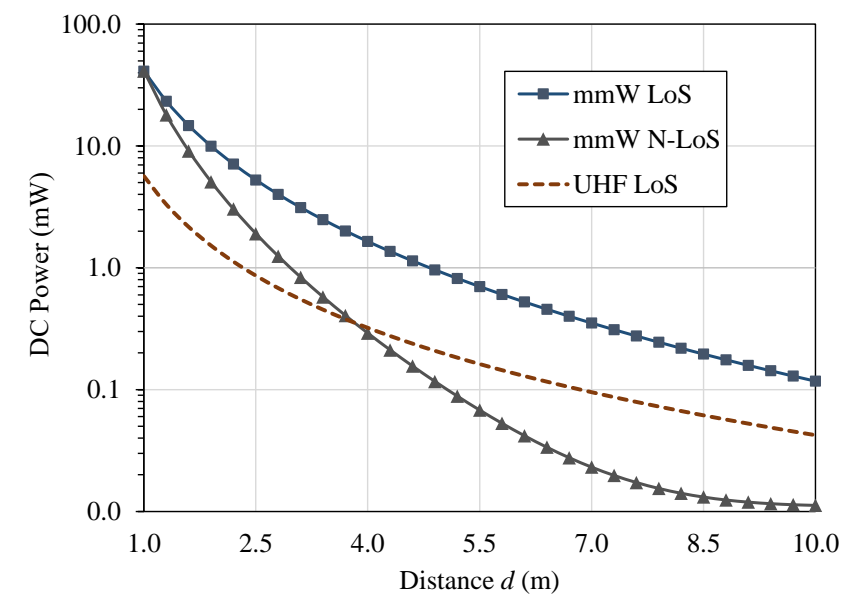

Fig. 16. Calculated received power by the $892 \mathrm{MHz}$ UHF patch and $13 \times 13$ $28 \mathrm{GHz}$ array, occupying the same area, based on empirical propagation losses.

antenna. The DC power Fig. 16 shows the calculated $P_{\mathrm{RX}}$ by the mmWave patch array and the single UHF patch. As the analysis in this section is based on the measured off-body gain of the patch, it is an accurate representation of the antenna's performance as a wearable wireless power receiver.

Despite the increased path losses, the lower PCE, and the empirical path loss exponent, for both the LoS and N-LoS cases, higher power can be delivered to the wearable receivers at $28 \mathrm{GHz}$ compared to $892 \mathrm{MHz}$. This demonstrates that body-centric WPT and Radio Frequency Energy Harvesting (RFEH) from an off-body source can achieve improved end-
TABLE II

TEXTILE-BASED LOS MMWAVE AND UHF WPT SUMMARY.

\begin{tabular}{|l|l|l|l|}
\hline & $\begin{array}{l}\mathrm{mmW} \\
\text { single }\end{array}$ & $\begin{array}{l}13 \times 13 \\
\mathrm{mmW} \text { array }\end{array}$ & $\begin{array}{l}\text { UHF single } \\
{[48]}\end{array}$ \\
\hline \hline Patch area $\left(\mathrm{mm}^{2}\right)$ & $9.0 \times 14$ & $187 \times 247$ & $190 \times 240$ \\
\hline$G(\mathrm{dBi})$ & 8.2 & - & 4.6 \\
\hline$P_{\mathrm{RX}}$ at $2 \mathrm{~m}(\mathrm{~mW})$ & 0.1 & 10.9 & 1.74 \\
\hline$P_{\mathrm{RX}}$ density $\left(\mathrm{mW} / \mathrm{m}^{2}\right)$ & 574 & 311 & 38.2 \\
\hline
\end{tabular}

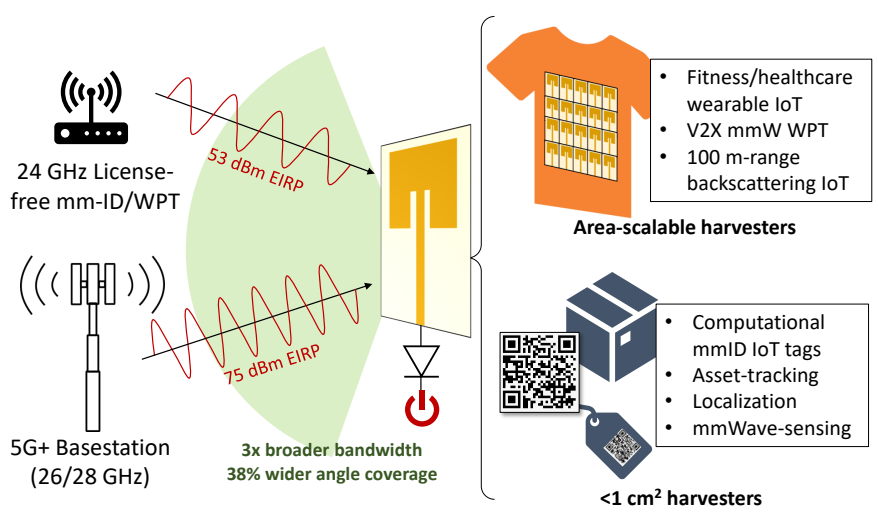

Fig. 17. Application scenarios of the proposed antenna in future mmWavepowered IoT devices.

to-end efficiency due to the smaller size of the antennas. Table II compares the power received by the mmWave and UHF antennas calculated using (5). The received DC power density is calculated using $P_{\mathrm{RX}} / A_{\text {Phys. }}$. Owing to the higher permissive EIRP and the compactness of the mmWave patch, the received power density is higher than that at UHF. Therefore, the large-area implementation can harvest up to $6.3 \times$ more power than the UHF rectenna occupying the same area. In addition, it is observed that despite the higher path-loss exponent in mmWave N-LoS propagation, higher power can still be harvested up to $3.5 \mathrm{~m}$ at $28 \mathrm{GHz}$, based on the proposed textile antenna.

\section{C. mmWave WPT in IoT Applications}

For a compact device, it was found that the proposed mmWave textile antenna will outperform its counterpart operating at $2.4 \mathrm{GHz}$ by $11 \mathrm{~dB}$ in terms of the channel gain, implying a 10-fold improvement in the received power. In the large-area scenario, a similar sized receiver can harvest $\mathrm{p}$ to $6 \times$ higher power than its similar-sized sub-1 GHz counterpart. Several recent WPT applications can benefit from the proposed low-cost, broadband, and high-efficiency antenna, as illustrated in Fig. 17.

For miniaturized receivers, high data-rate mmWave-ID circuits implemented in low-cost CMOS processes [53] can be integrated with the proposed antenna. Based on the free space path loss, $53 \mathrm{dBm}$ EIRP at $24 \mathrm{GHz}$, the proposed antenna's $8.2 \mathrm{dBi}$ gain, and the $6 \mathrm{dBm}$ sensitivity of [53], $10 \mathrm{MB} / \mathrm{s}$ battery-free communications can be sustained at approximately $60 \mathrm{~cm}$, while occupying less than $1 \mathrm{~cm}^{2}$ area and not requiring any external components. Similarly, the proposed low-cost antenna can power battery-free mmWave 
backscattering nodes such as [54]. Such highly-miniaturized ID tags can be adopted in future asset tracking and retail supply chains, enabling pervasive integration barcode tags. Furthermore, the proposed antenna maintains a lower profile than most wearable antennas [38]. Therefore, it can be applied to a wider variety of textile substrates, making it a better candidate for on-body IoT devices, such as RF-powered smart bandages [55].

Wearable fitness and healthcare trackers can rely on the proposed antenna for power harvesting as well as RF-based sensing. An on-body ethanol detection filtering rectenna was recently proposed at $2.4 \mathrm{GHz}$, and implemented on a lowloss polymer substrate [56]. Using the proposed antenna, the power harvesting front end can occupy $90 \%$ less area than the patch antenna in [56], while maintaining a high radiation efficiency on a wearable-friendly textile substrate. mmWave WPT from an off-body source can be utilized in future mmWave micro-cells in vehicle-to-anything (V2X) as well as indoor applications, where many IoT devices are expected to be powered simultaneously. Based on the measured sensitivity of practical rectifiers [4], a WPT range of $10 \mathrm{~m}$ can be achieved based on the FCC-specified $75 \mathrm{dBm}$ EIRP for the 5G mmWave bands [12], [17].

\section{CONCLUSions}

In this paper, the potential of mmWave WPT for powering wearable IoT devices has been investigated based on a novel broadband antenna. The proposed textile-based microstrip antenna represents the first realization of a broadband microstrip patch covering the the 26 and $28 \mathrm{GHz}$ bands on textiles. The wide bandwidth is enabled by operating at multiple resonant modes based on a simple structure. Moreover, the antenna's measured radiation properties show a radiation efficiency exceeding 50\% while maintaining a low profile, comparing favorably with recent textile and flexible mmWave antennas.

The antenna was analyzed for two WPT applications, a compact power receiver and a large-area array. The compact receiver shows $11 \mathrm{~dB}$ higher channel gain than similar sized microstrip antennas at $2.4 \mathrm{GHz}$. A large-area $13 \times 13$ patch rectenna array was evaluated numerically showing up to $10.3 \times$ and $5 \times$ higher power reception compared to a sub- $1 \mathrm{GHz}$ UHF patch antenna of the same size at $2 \mathrm{~m}$ separation from the transmitter, using empirical LoS and N-LoS propagation models, respectively. This shows that mmWave large-area harvesters can outperform their UHF counterparts based on DC combining within the license-free EIRP limits. It is concluded that textile-based patch antennas are suitable for RFEH applications in mmWave bands, despite the apparent increase in the path loss due to the smaller antenna effective area at mmWave bands. Furthermore, the use of non-linear physical analytical models for the power conversion is encouraged in analytical mmWave energy harvesting investigations, as opposed to fitted linear or quadratic models.

This work highlights the significance of mmWave WPT and the need for high-efficiency low-cost rectennas operating in mmWave bands, to allow WBANs to become energy-neutral using off-body mmWave WPT. High-efficiency rectifiers as well as DC power combining mechanisms are essential to allow mmWave antenna and rectenna arrays to surpass their UHF counterparts' energy harvesting performance.

\section{ACKNOWLEDGMENT}

The authors would like to thank the Communication Systems and Networks Group at the University of Bristol for providing access to the antenna measurement facilities, and Dr. C. R. Valenta from Georgia Tech Research Institute for allowing the reuse of the Matlab code used in the rectifier analysis. Data supporting this article will be made available from DOI: 10.5258/SOTON/D1931.

\section{REFERENCES}

[1] Z. Cai, Y. Zhou, Y. Qi, W. Zhuang, and L. Deng, "A millimeter wave dual-lens antenna for iot-based smart parking radar system," IEEE Internet of Things Journal, vol. 8, no. 1, pp. 418-427, 2021.

[2] R. Hussain, "Shared aperture slot-based sub-6 ghz and mm-wave iot antenna for 5g applications," IEEE Internet of Things Journal, pp. 1-1, 2021.

[3] A. Meredov, K. Klionovski, and A. Shamim, "Screen-Printed, Flexible, Parasitic Beam-Switching Millimeter-Wave Antenna Array for Wearable Applications," IEEE Open Journal of Antennas and Propagation, vol. 1, 2020.

[4] M. Wagih, A. S. Weddell, and S. Beeby, "Millimeter-Wave Power Harvesting: A Review," IEEE Open Journal of Antennas and Propagation, vol. 1 , pp. $560-578,2020$.

[5] N. Chahat, M. Zhadobov, S. A. Muhammad, L. L. Coq, and R. Sauleau, "60-GHz Textile Antenna Array for Body-Centric Communications," IEEE Trans. Antennas Propag., vol. 61 no. 4, pp. 1816 - 1824, 2013.

[6] N. Chahat, M. Zhadobov, L. L. Coq, and R. Sauleau, "Wearable Endfire Textile Antenna for On-Body Communications at $60 \mathrm{GHz}$," IEEE Antennas Wireless Propag. Lett., vol. 11, pp. 799 - 802, 2012.

[7] M. Ur-Rehman, N. A. Malik, X. Yang, Q. H. Abbasi, Z. Zhang, and N. Zhao, "A Low Profile Antenna for Millimeter-Wave Body-Centric Applications," IEEE Trans. Antennas Propag., vol. 65 no. 12, pp. 6329 - 6337, 2017.

[8] David M. Pozar, Microwave Engineering 4th Edition. NJ, United States: John Wiley \& Sons Inc, 2011.

[9] T. A. Khan, A. Alkhateeb, and R. W. Heath, "Millimeter Wave Energy Harvesting," IEEE Trans. Wireless Communications, vol. 15, 9, pp. 6048 $-6062,2016$

[10] J. Charthad, N. Dolatsha, A. Rekhi, and A. Arbabian, "System-Level Analysis of Far-Field Radio Frequency Power Delivery for mm-Sized Sensor Nodes," IEEE Trans. Circuits And Systems, vol. 63 no. 2, pp. $300-311,2016$

[11] M. Tabesh, N. Dolatsha, A. Arbabian, and A. M. Niknejad, "A PowerHarvesting Pad-Less Millimeter-Sized Radio," IEEE Journal of SolidState Circuits, vol. 50, 4, pp. 962 - 977, 2015.

[12] A. Eid, J. Hester, and M. Tentzeris, "5G as a wireless power grid." Sci Rep, vol. 11, p. 636, 2021.

[13] M. A. S. Tajin, C. E. Amanatides, G. Dion, and K. R. Dandekar, "Passive uhf rfid-based knitted wearable compression sensor," IEEE Internet of Things Journal, pp. 1-1, 2021.

[14] Y. Wang, J. Zhang, F. Peng, and S. Wu, "A glasses frame antenna for the applications in internet of things," IEEE Internet of Things Journal, vol. 6, no. 5, pp. 8911-8918, 2019.

[15] M. Wagih, G. S. Hilton, A. S. Weddell, and S. Beeby, "Dual-Band DualMode Textile Antenna/Rectenna for Simultaneous Wireless Information and Power Transfer (SWIPT)," IEEE Trans. Antennas Propag., 2021.

[16] D. Vital, S. Bhardwaj, and J. L. Volakis, "Textile Based Large Area RF-Power Harvesting System for Wearable Applications," IEEE Trans. Antennas Propag., vol. 68, no. 3, pp. 2323 - 2331, 2019.

[17] M. Wagih, G. S. Hilton, A. S. Weddell, and S. Beeby, "Broadband Millimetre-Wave Textile-based Flexible Rectenna for Wearable Energy Harvesting ," IEEE Trans. Microw Theory Techn, vol. 68 no. 11, pp. $4960-4972,2020$.

[18] O. L. A. Lpez, H. Alves, R. D. Souza, S. Montejo-Snchez, E. M. G. Fernndez, and M. Latva-Aho, "Massive wireless energy transfer: Enabling sustainable iot toward 6g era," IEEE Internet of Things Journal, vol. 8 , no. 11 , pp. $8816-8835,2021$. 
[19] T. X. Tran, W. Wang, S. Luo, and K. C. Teh, "Nonlinear Energy Harvesting for Millimeter Wave Networks With Large-Scale Antennas," IEEE Trans. Veh. Technol., vol. 67 no. 10, pp. 9488 - 9498, 2018.

[20] M. Wagih, O. Cetinkaya, B. Zaghari, A. S. Weddell, and S. Beeby, "Real-World Performance of Sub-1 GHz and 2.4 GHz Textile Antennas for RF-Powered Body Area Networks," IEEE Access, vol. 8, pp. 133746 - 133756, 2020.

[21] M. Wagih, A. S. Weddell, and S. Beeby, "Omnidirectional DualPolarized Low-Profile Textile Rectenna with over 50\% Efficiency for Sub- $\mu \mathrm{W} / \mathrm{cm}^{2}$ Wearable Power Harvesting," IEEE Trans. Antennas Propag., vol. Early Access, 2020.

[22] D. Ghodgaonkar, O. Gandhi, and M. Iskander, "Complex permittivity of human skin in vivo in the frequency band 26.5-60 GHz," in IEEE Antennas and Propagation Society International Symposium, 2000.

[23] N.-W. Liu, L. Zhu, W.-W. Choi, and J.-D. Zhang, "A Low-Profile Differentially Fed Microstrip Patch Antenna With Broad Impedance Bandwidth Under Triple-Mode Resonance," IEEE Antennas Wireless Propag. Lett., vol. 17 no. 8, pp. 1478 - 1482, 2018.

[24] J. Yin, Q. Wu, C. Yu, H. Wang, and W. Hong, "Broadband Symmetrical E-Shaped Patch Antenna With Multimode Resonance for 5G MillimeterWave Applications," IEEE Trans. Antennas Propag., vol. 67 no. 7, pp. $4474-4483,2019$.

[25] C. A. Balanis, "Antenna Theory: Analysis and Design. Third Edition," Wiley Interscience, pp. 830 - 833, 2005.

[26] 5G Spectrum Positions. [Online]. Available: www.gsma.com/spectrum/wp-content/uploads/2019/02/5G-SpectrumPositions-InfoG.pdf

[27] N. Chahat, M. Zhadobov, L. L. Coq, S. I. Alekseev, and R. Sauleau, "Characterization of the Interactions Between a 60-GHz Antenna and the Human Body in an Off-Body Scenario," IEEE Trans. Antennas Propag., vol. 60 no. 12 , pp. 5958 - 5965, 2012.

[28] A. R. Guraliuc, M. Zhadobov, O. D. Sagazan, and R. Sauleau, "Solid Phantom for Body-Centric Propagation Measurements at $60 \mathrm{GHz}$," IEEE Trans. Microw. Theory Techn., vol. 62 no. 6, pp. 1373 - 1380, 2014.

[29] M. Wagih, Y. Wei, A. Komolafe, R. Torah, and S. Beeby, "Reliable UHF Long-Range Textile-Integrated RFID Tag Based on a Compact Flexible Antenna Filament," Sensors, vol. 20 (12), p. 3435, 2020.

[30] M. Wagih, A. S. Weddell, and S. Beeby, "Millimeter-Wave Textile Antenna for On-Body RF Energy Harvesting in Future 5G Networks," in 2019 IEEE Wireless Power Transfer Conference (WPTC), 2019.

[31] A. Eid, J. Hester, and M. M. Tentzeris, "A Scalable High-Gain and Large-Beamwidth mm-Wave Harvesting Approach for 5G-powered IoT," in 2019 IEEE MTT-S International Microwave Symposium (IMS), 2019.

[32] S. Shen, Y. Zhang, C.-Y. Chiu, and R. Murch, "An Ambient RF Energy Harvesting System Where the Number of Antenna Ports Is Dependent on Frequency," IEEE Trans. Microw. Theory Techn., vol. 67 no. 9, pp. $3821-3832,2019$

[33] S. Ladan, A. B. Guntupalli, and K. Wu, "A High-Efficiency $24 \mathrm{GHz}$ Rectenna Development Towards Millimeter-Wave Energy Harvesting and Wireless Power Transmission," IEEE Trans. Circuits And Systems, vol. 61, 12, pp. $3358-3366,2014$.

[34] M. Wagih, A. S. Weddell, and S. Beeby, "Rectennas for RF Energy Harvesting and Wireless Power Transfer: a Review of Antenna Design [Antenna Applications Corner]," IEEE Antennas Propag. Mag., vol. 62 no. 5 , pp. $95-107,2020$.

[35] K. Turbic, L. M. Correia, and M. Beko, "A Channel Model for Polarized Off-Body Communications With Dynamic Users," IEEE Trans. Antennas Propag., vol. 67 no. 11, pp. 7001 - 7013, 2019.

[36] H. Sun, H. He, and J. Huang, "Polarization-Insensitive Rectenna Arrays With Different Power Combining Strategies," IEEE Antennas Wireless Propag. Lett., vol. 19, 3, pp. 492 - 496, 2020.

[37] D. L. Paul, H. Giddens, M. G. Paterson, G. S. Hilton, and J. P. McGeehan, "Impact of Body and Clothing on a Wearable Textile Dual Band Antenna at Digital Television and Wireless Communications Bands," IEEE Trans. Antennas Propag., vol. 61 no. 4, pp. 2188 - 2194 2013.

[38] S.-E. Adami, P. Proynov, G. S. Hilton, G. Yang, C. Zhang, D. Zhu, Y. Li, S. P. Beeby, I. J. Craddock, and B. H. Stark, "A Flexible 2.45GHz Power Harvesting Wristband With Net System Output From -24.3 $\mathrm{dBm}$ of RF Power," IEEE Trans. Microw. Theory Techn., vol. 66 no. 1, pp. 380-395, 2018.

[39] H. Chen, Y. Shao, Y. Zhang, C. Zhang, and Z. Zhang, "A Low-Profile Broadband Circularly Polarized mmWave Antenna With Special-Shaped Ring Slot," IEEE Antennas Wireless Propag. Lett., vol. 18, 7, pp. 1492 $-1496,2019$
[40] B. S. Cook, B. Tehrani, J. R. Cooper, and M. M. Tentzeris, "Multilayer Inkjet Printing of Millimeter-Wave Proximity-Fed Patch Arrays on Flexible Substrates," IEEE Antennas Wireless Propag. Lett., vol. 12, pp. $1351-1354,2013$.

[41] J. Bito, V. Palazzi, J. Hester, R. Bahr, F. Alimenti, P. Mezzanotte, L. Roselli, and M. M. Tentzeris, "Millimeter-wave ink-jet printed RF energy harvester for next generation flexible electronics," in 2017 IEEE Wireless Power Transfer Conference (WPTC), 2017.

[42] T.-W. Yoo and K. Chang, "Theoretical and experimental development of 10 and $35 \mathrm{GHz}$ rectennas," IEEE Trans. Mirow Theory Techn., vol. 40, 6, pp. 1259 - 1266, 1992.

[43] C. R. Valenta, M. M. Morys, and G. D. Durgin, "Theoretical EnergyConversion Efficiency for Energy-Harvesting Circuits Under PowerOptimized Waveform Excitation," IEEE Trans. Microw. Theory Techn., vol. 63 no. 5 , pp. $1758-1767,2015$.

[44] S. Hemour, C. H. P. Lorenz, and K. Wu, "Small-footprint wideband $94 \mathrm{GHz}$ rectifier for swarm micro-robotics," in 2015 IEEE MTT-S International Microwave Symposium, 2015.

[45] H. Mei, X. Yang, B. Han, and G. Tan, "High-efficiency microstrip rectenna for microwave power transmission at Ka band with low cost," IET Microwave Antennas and Propagation, vol. 10 no. 15, pp. 16481655,2016

[46] Y. Wang, X.-X. Yang, G.-N. Tan, and S. Gao, "Study on millimeterwave siw rectenna and arrays with high conversion efficiency," IEEE Transactions on Antennas and Propagation, pp. 1-1, 2021.

[47] A. Harutyunyan and M. Landwehr, "A 61-ghz rectifier using internal voltage cancellation and body-biasing techniques in 22-nm fdsoi," IEEE Microwave and Wireless Components Letters, vol. 31, no. 6, pp. 764767, 2021

[48] G. Monti, L. Corchia, and L. Tarricone, "UHF Wearable Rectenna on Textile Materials," IEEE Trans. Antennas. Propag., vol. 61, 7, pp. 3869 - 3873, 2013.

[49] Y. Huo, X. Dong, and W. Xu, "5g cellular user equipment: From theory to practical hardware design," IEEE Access, vol. 5, 2017.

[50] FCC. Revision of Part 15 of the Commission's Rules to Permit Unlicensed National Information Infrastructure (U-NII) Devices in the $5 \mathrm{GHz}$ Band. [Online]. Available: https://www.fcc.gov/document/5-ghzu-nii-ro

[51] C. A. Balanis, "Antenna Theory: Analysis and Design. Third Edition," Wiley Interscience, pp. $84-85,2005$.

[52] G. R. MacCartney, S. Deng, and T. S. Rappaport, "Indoor Office Plan Environment and Layout-Based mmWave Path Loss Models for $28 \mathrm{GHz}$ and $73 \mathrm{GHz}$," in 2016 IEEE 83rd Vehicular Technology Conference (VTC Spring), 2016

[53] P. Burasa, N. G. Constantin, and K. Wu, "High-Data-Rate Single-Chip Battery-Free Active Millimeter-Wave Identification Tag in 65-nm CMOS Technology," IEEE Trans. Microw. Theory Techn., vol. 64 no. 7, pp. $2294-2303,2016$

[54] A. Eid, J. Hester, and E. M. Tentzeris, "Rotman Lens-Based Wide Angular Coverage and High Gain Semi-Passive Architecture for UltraLong Range mm-wave RFIDs," IEEE Antennas Wireless Propag. Lett., vol. 19 no. 11 , pp. 1943 - 1947, 2020.

[55] P. Escobedo, M. Bhattacharjee, F. Nikbakhtnasrabadi, and R. Dahiya, "Smart bandage with wireless strain and temperature sensors and batteryless nfc tag," IEEE Internet of Things Journal, vol. 8, no. 6, pp. 5093-5100, 2021.

[56] F. Benassi, G. Paolini, D. Masotti, and A. Costanzo, "A wearable flexible energy-autonomous filtenna for ethanol detection at $2.45 \mathrm{ghz}$," IEEE Transactions on Microwave Theory and Techniques, pp. 1-1, 2021 